\title{
Enhanced Sensitivity of Surface Plasmon Resonance Biosensor Functionalized with Doped Polyaniline Composites for the Detection of Low-Concentration Acetone Vapour
}

\author{
Fahad Usman $\mathbb{D}^{1,2}$ John Ojur Dennis, ${ }^{1}$ Khe Cheng Seong, ${ }^{1}$ Abdelaziz Yousif Ahmed, ${ }^{3}$ \\ Thomas L. Ferrell, ${ }^{4}$ Yap Wing Fen, ${ }^{5,6}$ Amir Reza Sadrolhosseini, ${ }^{6}$ \\ Olumide Bolarinwa Ayodele, ${ }^{7}$ Fabrice Meriaudeau, ${ }^{8}$ and Aminu Saidu ${ }^{9}$ \\ ${ }^{1}$ Department of Fundamental and Applied Sciences, Universiti Teknologi PETRONAS, Seri Iskandar, 32610 Perak, Malaysia \\ ${ }^{2}$ Department of Physics, Al-Qalam University, Katsina, Katsina PMB 2137, Nigeria \\ ${ }^{3}$ Department of Electrical and Electronic Engineering, Universiti Teknologi PETRONAS, Seri Iskandar, 32610 Perak, Malaysia \\ ${ }^{4}$ Dept of Physics and Astronomy, University of Tennessee, 401 Nielsen Physics Building and Joint Institute for Materials Research 1408 \\ Circle Drive Room 2192641 Osprey Way, Knoxville, TN 37996 Tennessee, USA \\ ${ }^{5}$ Department of Physics, Universiti Putra Malaysia, Serdang, 43400 Selangor, Malaysia \\ ${ }^{6}$ Institute of Advanced Technology, Universiti Putra Malaysia, Serdang, 43400 Selangor, Malaysia \\ ${ }^{7}$ Micro and Nanofabrication Department, International Iberian Nanotechnology Laboratory, 4715-330 Braga, Portugal \\ ${ }^{8}$ ImViA EA 7535, Team IFTIM, Université de Bourgogne, 21000 Dijon, France \\ ${ }^{9}$ Department of Physics, Faculty of Science, Usmanu Danfodiyo University Sokoto, PMB 2346 Sokoto, Nigeria
}

Correspondence should be addressed to Fahad Usman; fahatu11@gmail.com

Received 5 August 2019; Accepted 16 October 2019; Published 20 November 2019

Academic Editor: Sachin K. Srivastava

Copyright ( $) 2019$ Fahad Usman et al. This is an open access article distributed under the Creative Commons Attribution License, which permits unrestricted use, distribution, and reproduction in any medium, provided the original work is properly cited.

\begin{abstract}
PANI/chitosan composite and a ternary composite comprising of PANI, chitosan, and reduced graphene oxide have been successfully synthesised and characterised using FTIR and UV-VIS spectroscopy. Optical constants of the composites were extracted from the UV-VIS spectra. The extracted parameters were applied in the simulation of a surface plasmon resonance (SPR) biosensor functionalised with PANI/chitosan and ternary composites. The aim was to explore the applicability of the composite-based SPR sensor in the detection of low-concentration acetone vapour within the range of $1.8 \mathrm{ppm}-5.0 \mathrm{ppm}$ for diabetes monitoring and screening. The functionalization of the SPR sensor with the PANI/chitosan and the ternary composites shows promising application of the sensor in the detection of acetone vapour at a low concentration down to less than $0.5 \mathrm{ppm}$. The maximum sensitivity values of about 60 and 180 degree/refractive index change were observed for PANI/chitosan and ternary composite sensing layers, respectively, in comparison with the bare gold-based SPR which shows no response up to $10 \mathrm{ppm}$ concentration of acetone vapour in air. In addition, the two sensing layers show good selectivity to acetone vapour compared to ethanol, methanol, and ammonia. The response in the case of ternary composite shows better linearity with a correlation coefficient of 1.0 compared to PANI/chitosan- and gold-based SPR layers with 0.9999 and 0.9997 , respectively.
\end{abstract}

\section{Introduction}

The interaction between light waves and conduction electrons at the interface of metal and dielectric media produces quanta of longitudinal surface waves. The waves are called surface plasmons and are confined at a smaller wavelength along the surface of the metal (plasmonic material) in contact with the dielectric interface [1-3]. Surface plasmon resonance (SPR) happens when energy is exchanged between the light waves and surface plasmons. The incident angle of the light at which nearly complete attenuation of the reflected light occurs is called the SPR angle, and it is a function of the optical properties of the metal, dielectric medium, and any adsorbate on the metal. This dependency is exploited in 
SPR biosensing [3, 4]. SPR-based sensors feature high sensitivity, real-time noninvasive measurement, label-free measurement, and nonrequirement of electrodes [1, 4].

Gold is widely used as the plasmonic material in SPR biosensors due to its high SPR shift feature (allowing high sensitivity) and chemical stability [1]. However, despite these important features, detection of volatile compounds and other gases (few hundreds of Daltons) in low concentrations is difficult to achieve. This is due to their poor interaction with gold surface $[5,6]$. But detection and monitoring of the molecules of such volatile compounds are required in many aspects such as the environmental monitoring of toxic gases that are released through unintended processes as well as the quality control and industrial monitoring of many chemical products [7-9]. In addition, some of these molecules can serve as disease biomarkers. An example is the use ammonia in monitoring kidney complications and acetone as a diabetes biomarker [10-14].

Exhaled breath acetone can be used as a diabetes biomarker because of its high correlation with blood glucose (conventional diabetes biomarker) [15]. The concentration of acetone in the human body is generally very low (0.1 ppm-0.8 ppm), although it can be higher in the case of metabolism disorders, including diabetes mellitus (DM) (1.8 ppm-5.0 ppm) [16-18]. Detection of such very low concentration has been achieved by chemiresistor-based sensors that mostly employ metal oxide semiconductors [19, 20]. However, the contact resistance of the sensors' electrodes also contributes to the overall resistance of the device. This leads to unreliable detection and poor selectivity, as little information other than resistance can be obtained [21]. Furthermore, metal oxide semiconductor sensors operate at a high temperature $[19,22-25]$ resulting to an increased energy consumption and unfavourable detection. SPR sensors are not subject to these effects.

SPR-based sensors have been successfully applied in the detection of some volatile compounds and gases like benzene, ammonia, chloroform, ethanol, methanol, ethyl benzene, 2-propanol, toluene, and acetone vapours [8, 26-32]. The performance of the sensors has been improved through various design modifications which include the incorporation of nanoparticles of different morphologies [33-35], bimetallic layers [2, 36-39], porous gold [40-43], graphene layer on gold $[5,44]$, conducting and insulating polymer coatings [26, 45, 46], and metal oxide coatings [47, 48]. However, SPR detection of acetone vapour has not been optimized for suitable applicability in diabetes monitoring and screening [32].

Conducting polymers, particularly, polyaniline, have been used as an active layer in gas sensors since early 1980s [49]. In contrast to metal oxide-based sensors, polyanilinebased sensors are operated at room temperature [21]. Polyaniline is also widely researched for its easy synthesis, high sensitivity even at room temperature, short response time, good mechanical properties enabling facile fabrication, high surface area to volume ratio, and high surface tension. Furthermore, chitosan has demonstrated ppm-level detection of acetone vapour based on a different means of detection [50]. Graphene-based SPR sensors are also well known at improving sensitivity and selectivity due to graphene's unique physical, optical, and electrical properties [51-53]. Additionally, adsorption of gas molecule onto the surface of an organic material (active sensing layer) can alter its structural, electrical, or optical properties. Better sensitivity and selectivity are normally expected with possible solvation effect/and potential of hydrogen bond formation. Fortunately, Hildebrand and Hansen solubility parameters of polyaniline and acetone are closer than many other similar vapours [54]. In addition, both polyaniline and chitosan can form a hydrogen bond with the $\mathrm{CO}$ group from acetone $[54,55]$. Furthermore, graphene has been described as among the best adsorbent material for carbon-based biomolecules [56].

In this work, we have synthesised p-toluene sulfonicdoped polyaniline (PANI)/chitosan composite and a ternary composite comprising of PANI, chitosan, and reduced graphene oxide (RGO). The composites were characterised by ultra violet-visible spectroscopy (UV-VIS) and Fourier transform infrared spectroscopy (FTIR). Furthermore, the optical properties of the composites were extracted from their respective optical spectra (UV-VIS). The applicability of the PANI/chitosan and the ternary composites in SPR sensing was then investigated and compared with conventional gold-based SPR sensor using mathematical modeling and simulation. Our aim was to investigate the potential of the PANI/chitosan and the ternary composite-based SPR biosensors for utilization in the detection of low concentration of acetone vapour for diabetes monitoring and screening.

\section{Materials and Methods}

2.1. Materials. Aniline monomer (Aniline 99\%), p-toluene sulfonic acid (PTSA), ammonium peroxodisulfate (APS) (98\%), graphene oxide (GO), acetic acid, hydrazine monohydrate $(>99 \%)$, and chitosan were all supplied by Avanti chemicals supply from Merck and Sigma-Aldrich. All the chemicals were analytical grade. RGO was obtained by hydrazine reduction.

\subsection{Synthesis of PANI Composites and Characterisation}

2.2.1. PANI/Chitosan Composites. The synthesis of PANI/ chitosan composites is in accordance with some previous works $[57,58]$. In a typical synthesis, $0.5 \mathrm{~g}$ of chitosan was dissolved in $50 \mathrm{ml}$ of aqueous acetic acid $(2 \% v / v)$, and the solution was stirred for 24 hours at room temperature. To this mixture, about $30 \mathrm{ml}$ of $0.1 \mathrm{M}$ of aniline (dissolved in $0.4 \mathrm{M}$ PTSA) was added and stirred for $15 \mathrm{~min}$ to form a homogenous solution. The resultant mixture was cooled to less than $5^{\circ} \mathrm{C}$. In order to initialised the polymerisation process, $10 \mathrm{ml}$ of $0.15 \mathrm{M}$ solution of APS was prepared in $0.1 \mathrm{M}$ PTSA and added to the above homogenous solution drop by drop with constant stirring at $0^{\circ} \mathrm{C}-5^{\circ} \mathrm{C}$. After that, the reaction mixture was kept under constant stirring for 6 hours. A greenish-black precipitate was obtained. The precipitate was filtered and washed (with water and ethanol) until the filtrate becomes colourless. The final composite was dried at $60^{\circ} \mathrm{C}$ for 24 hours. 
2.2.2. Ternary Composites. Based on the previous literatures $[59,60]$, a ternary composite was synthesised. In a typical synthesis, $1 \mathrm{~g}$ of chitosan was dissolved in $100 \mathrm{ml}$ of aqueous acetic acid $(2 \% v / v)$, and the solution was stirred for 24 hours at room temperature. A separate homogeneous dispersion of $100 \mathrm{mg}$ RGO in $45 \mathrm{ml}$ of $0.4 \mathrm{M}$ solution of PTSA was prepared by ultrasonication for 2 hours. The two components were mixed under continuous stirring. To the resulting mixture, about $50 \mathrm{ml}$ of $0.1 \mathrm{M}$ of aniline (dissolved in $0.4 \mathrm{M}$ PTSA) was added and stirred for 15 minutes to form a homogenous dispersion. $0.15 \mathrm{M}$ APS solution was then added to the dispersion drop by drop with constant stirring at $0^{\circ} \mathrm{C}-5^{\circ} \mathrm{C}$. The reaction mixture was kept under constant stirring for an additional 6 hours. A greenish-black precipitate was obtained. It was then filtered and washed with water and ethanol until the filtrate becomes colourless. The final composite was dried at $60^{\circ} \mathrm{C}$ for 24 hours.

2.2.3. Characterisation. The successful synthesis of the PANI composites was confirmed by FTIR spectroscopy (PerkinElmer, Spectrum One) in the $4000-400 \mathrm{~cm}^{-1}$ range. UV-VIS spectroscopy of the composites' thin films was also carried out in order to further confirm the synthesis and for optical constant measurements. The thickness measurement was conducted using surface roughness tester, SV-mutitoyo-3000.

\subsection{Optical Constants and SPR Studies of the PANI Composites}

2.3.1. Optical Constant of the PANI/Chitosan and the Ternary Composites. The optical constants of the composites were extracted from the UV-VIS spectra of the thin film of the respective composites. The thin films were deposited from the dispersion concentration of $0.015 \mathrm{~g} / \mathrm{ml}$. Deionised water was used as solvent in the case of the PANI/chitosan and the ternary composites while 1-Methyl-2-pyrrolidinone (NMP) was used in the case of PANI and PANI/RGO. The process was complemented by constant magnetic stirring for 3 hours at a $350 \mathrm{rpm}$ test. Later, the resulting dispersions were spin coated using POLOS ${ }^{\mathrm{TM}}$ spin coater at $1500 \mathrm{rpm}$.

The extraction of the optical constants was based on the well-known relationship between the reflectance $(R)$, transmittance $(T)$, and absorbance $(A)$ spectral data [61]. This is shown in equation (1). The optical absorption based on Beer's law, transmittance, and absorption coefficient $(\alpha)$ of the composites were also calculated using equations (2), (3), and (4), respectively [61, 62].

$$
\begin{array}{r}
R+T+A=1 . \\
I(d)=I_{0} e^{-\alpha d},
\end{array}
$$

where $I(d)$ is the intensity at depth of thickness $d, I_{0}$ is intensity at zero thickness, and $\alpha$ is absorption coefficient.

$$
T=(1-R)^{2} e^{-\alpha d}
$$

$$
\alpha=\frac{2.303 A}{d}
$$

However, the extinction coefficient, $k$ (imaginary part refractive index) is related to $\alpha$ by equation

$$
k=\frac{\alpha \lambda}{4 \pi}
$$

where $\lambda$ is the wavelength of the light waves $(633 \mathrm{~nm}$ in this case).

Based on all these above equations ((1)-(5)), the real part of refractive indices, $n$ of the composites were evaluated from the Fresnel equation (equation (6))-based reflectivity measurement of the composites using equation (7) [61, 62]

$$
\begin{aligned}
& R=\left|\frac{\tilde{n}-1}{\tilde{n}+1}\right|^{2}=\frac{(n-1)^{2}+k^{2}}{(n+1)^{2}+k^{2}} . \\
& n=\left[\frac{1+R}{1-R}\right]+\sqrt{\frac{4 R}{(1-R)^{2}}}-k^{2} .
\end{aligned}
$$

Furthermore, the complex dielectric constant values of the composites were evaluated from the $n$ and $k$ values using the ff. equations [61]

$$
\begin{aligned}
& \varepsilon_{1}=n^{2}-k^{2} . \\
& \varepsilon_{2}=2 n k,
\end{aligned}
$$

where $\varepsilon_{1}$ and $\varepsilon_{2}$ are real part and imaginary part dielectric constants, respectively.

2.3.2. SPR Studies of the Composites. The design of a surface plasmon-based sensors is guided by the knowledge of the penetration depth of a surface plasmon wave. It is defined as the distance from the interface of metal-dielectric at which the amplitude of the field becomes $1 / e$ of the value at the interface [1]. The penetration depth in the dielectric gives us a measure of the length over which the surface plasmon is sensitive to the changes in the refractive index of the dielectric medium, while the penetration depth into metal gives us an idea of the thickness of the metal film required for the coupling of light incident from the other interface of the metal film [1]. In this study, the dielectric constant value of gold from literature and that of the composites was substituted in equations (10) and (11) in order to find the penetration depth through the gold $\left(\delta_{m}\right)$ and the composite materials $\left(\delta_{d}\right)$ adjacent to the gold film, respectively [26].

$$
\begin{gathered}
\delta_{m}=\frac{\lambda_{0}}{2 \pi}\left[\frac{\varepsilon_{m}^{\prime}+\varepsilon_{d}}{\left(\varepsilon_{m}^{\prime}\right)^{2}}\right]^{1 / 2} . \\
\delta_{d}=\frac{\lambda_{0}}{2 \pi}\left[\frac{\varepsilon_{m}^{\prime}+\varepsilon_{d}}{\varepsilon_{d}}\right]^{1 / 2},
\end{gathered}
$$




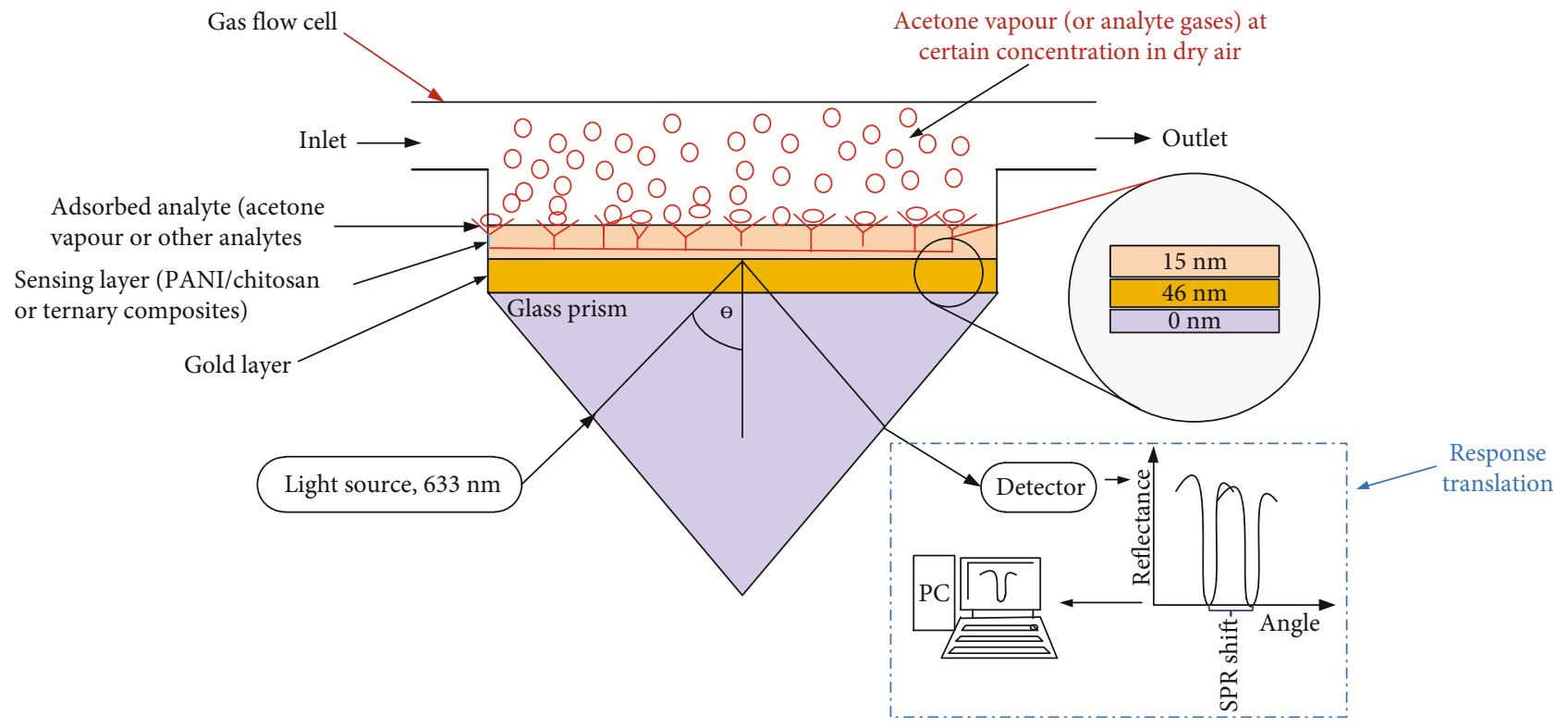

FIGURE 1: Diagram of the proposed SPR sensor based on PANI/chitosan and ternary composites.

where $\lambda_{0}$ is the free space wavelength and $\varepsilon_{m}^{\prime}$ and $\varepsilon_{d}$ are the real part dielectric constant of gold and the material adjacent to the gold, respectively.

The design was further optimized using 5 different light sources, namely red light at $633 \mathrm{~nm}$, yellow light at $589 \mathrm{~nm}$, green light at $546 \mathrm{~nm}$, violet light at $441 \mathrm{~nm}$, and blue light at $436 \mathrm{~nm}$ (G-Line). The sketch diagram of the sensing device is shown in Figure 1.

The system is comprised of $\mathrm{He}-\mathrm{Ne}$ laser light $\left(\lambda_{0}=633 \mathrm{~nm}\right)$, SF11 prism $\left(n_{1}=1.7786\right.$ at $\left.633 \mathrm{~nm}\right)$, Au $\left(d_{2}=46 \mathrm{~nm}, n_{2}=0.19404+3.5934 i\right)[63,64]$, PANI/chitosan composite or ternary composites $\left(d_{3}=15 \mathrm{~nm}\right)$, and dry air environment. Using equations (5) and (7), the complex refractive indices $\left(n_{3}\right)$ of PANI/chitosan and ternary composites at $633 \mathrm{~nm}$ were found to be $1.803+0.318 i$ and $2.526+0.432 i$, respectively. The refractive index of dry air $\left(n_{4}\right)$ at temperature $(t)=25^{\circ} \mathrm{C}$ and pressure $(p)=101325 \mathrm{~Pa}$ was found to be 1.000263719 using the ff. equation $[65,66]$

$$
n_{4}-1=\left(n_{s}-1\right) \times \frac{p\left[1+p(60.1-0.972 t) \times 10^{-10}\right]}{96095.43(1+0.003661 t)},
$$

where $n_{s}$ is the refractive of standard air at $633 \mathrm{~nm}, 15^{\circ} \mathrm{C}$, and 101325 with $0.045 \%$ by volume of carbon dioxide (1.000272874).

To explore the response linearity of the proposed sensor, the transfer matrix method (TMM) was applied in MATLAB environment to generate SPR curves. The system was considered to be composed of $N$ layers with dielectric constants and thicknesses $\varepsilon_{\mathrm{i}}$ and $d_{\mathrm{i}}$, respectively, placed between SF11 prism with dielectric constant $\varepsilon_{\mathrm{p}}$ and air medium with dielectric constant $\varepsilon_{\text {air }}$. The system also assumed a fixed thickness refractive index change of 0.005 units due to the presence of analyte of certain concentration. The complex reflection coefficient $\left(r_{p}\right)$ for the p-polarised incident light was therefore described using equation [3]

$r_{p}(\alpha)=\frac{\left(M_{11}+M_{12}\left(k_{\mathrm{y}, \mathrm{air}} / \varepsilon_{\mathrm{air}}\right)\right)\left(k_{y, p} / \varepsilon_{p}\right)-\left(M_{21}+M_{22}\left(k_{\mathrm{y}, \mathrm{air}} / \varepsilon_{\mathrm{air}}\right)\right)}{\left(M_{11}+M_{12}\left(k_{\mathrm{y}, \mathrm{air}} / \varepsilon_{\mathrm{air}}\right)\right)\left(k_{y, p} / \varepsilon_{p}\right)+\left(M_{21}+M_{22}\left(k_{\mathrm{y}, \mathrm{air}} / \varepsilon_{\mathrm{air}}\right)\right)}$,

where $M$ represents the transfer matrix:

$$
\begin{aligned}
& M=M_{1} \cdot M_{2} \cdots \cdots M_{N} \text {, } \\
& M_{i}=\left(\begin{array}{cc}
\cos \left(k_{y, i} d_{i}\right) & \frac{-j \varepsilon_{i}}{k_{y, i}} \sin \left(k_{y, i} d_{i}\right) \\
\frac{-j k_{y, i}}{\varepsilon_{i}} \sin \left(k_{y, i} d_{i}\right) & \cos \left(k_{y, i} d_{i}\right)
\end{array}\right) \text {, }
\end{aligned}
$$

where $k_{y}$ is the wave vector perpendicular to the interface.

The angular dependence of $r_{p}$ is contained in the $k_{y, i}$, and the reflectance was obtained from equation

$$
R_{p}=\left|r_{p}\right|^{2}
$$

For the SPR simulation of the detection of low concentration of acetone vapour in dry air for diabetes monitoring, the Arago-Biot mixing formula (equation (17)) was applied for the estimation of the refractive index of $0.5 \mathrm{ppm}, 2 \mathrm{ppm}$, $4 \mathrm{ppm}, 6 \mathrm{ppm}, 8 \mathrm{ppm}$, and $10 \mathrm{ppm}$ concentration of acetone vapour in dry air [67].

$$
n_{\text {acetone }(\mathrm{ppm})}=\phi_{\text {air }} n_{\text {air }}+\phi_{\text {acetone }} n_{\text {acetone }}
$$

where $\phi_{\text {acetone }}$ is the $n$-millionth fraction by volume of acetone vapour, $n=0.5,2,4,6,8$, and 10 for $0.5 \mathrm{ppm}, 2 \mathrm{ppm}$, 


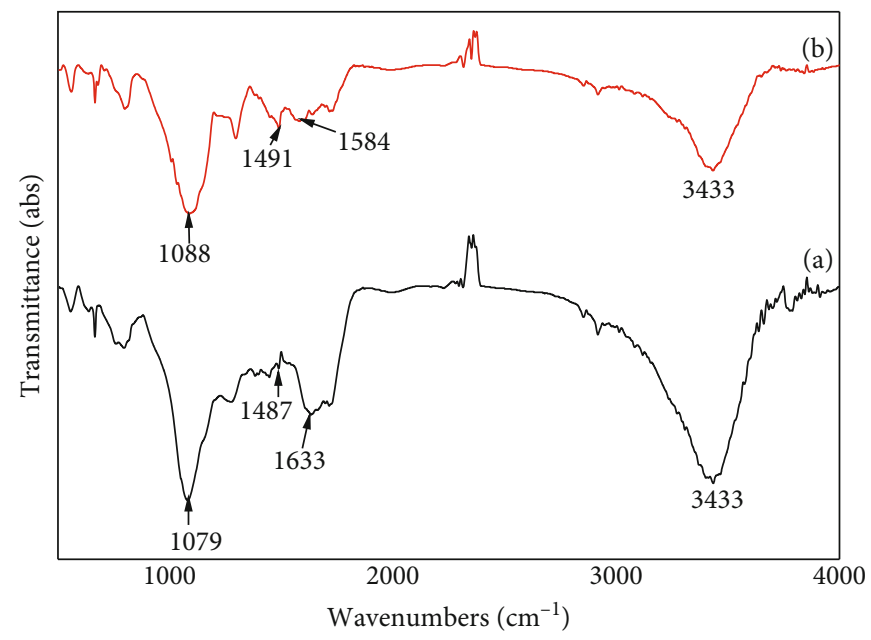

FIgURE 2: FTIR spectra of (a) PANI/chitosan and (b) ternary composites.

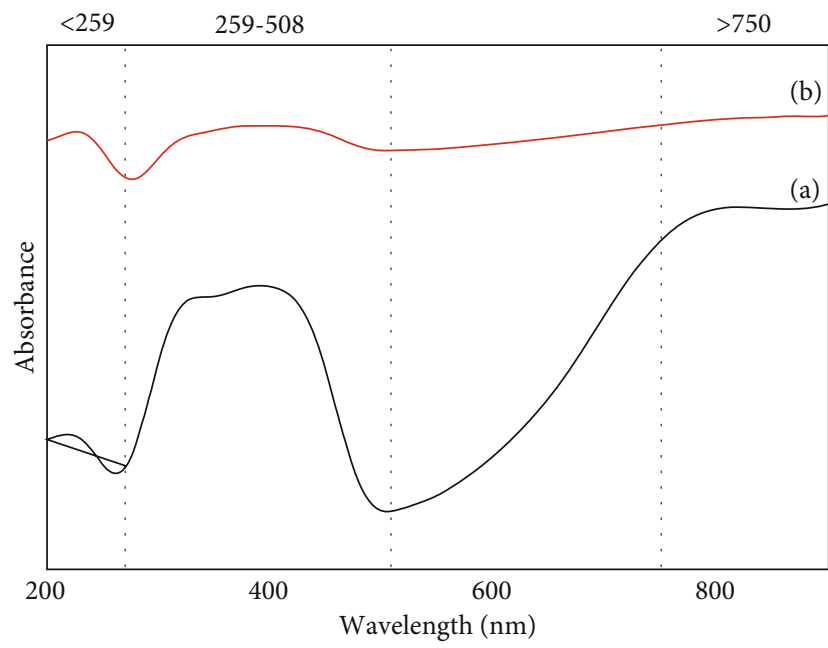

FIGURE 3: UV-VIS spectra of (a) PANI/chitosan and (b) ternary composites.

$4 \mathrm{ppm}, 6 \mathrm{ppm}, 8 \mathrm{ppm}$, and $10 \mathrm{ppm}$ of acetone, respectively. Also, $\phi_{\text {air }}=1-\phi_{\text {acetone }} \cdot n_{\text {air }}$ and $n_{\text {acetone }}$ are the refractive index of dry air and acetone vapour, respectively.

Furthermore, the detection of such low concentration of acetone vapour was simulated based on the following assumptions. The thickness of gold was considered to be constant during the detection process due to its lack of good adsorption capability for gases [6]. However, the thicknesses and the refractive indices of PANI/chitosan and ternary composites were increased by 0.0005 and $0.1 \mathrm{~nm}$ units, respectively. This is due to potential adsorption, solvation, and swelling effect of PANI and chitosan [26, 30, 54, 68-70]. Equation (18) was applied in comparing the sensitivity of the SPR sensors based on gold, PANI/chitosan, and ternary composite [3]. The refractive index change, $\Delta n$, of the sensing layer was considered in the case of PANI/chitosan and ternary composites because the contributions from the gas-phase atop the polymer are not anticipated to substan-
TABLE 1: Assignment of FTIR peaks in PANI/chitosan and ternary composites.

\begin{tabular}{lcc}
\hline & \multicolumn{2}{c}{ Absorption frequencies $\left(\mathrm{cm}^{-1}\right)$} \\
& PANI/chitosan & Ternary \\
\hline NH stretching & 3433 & 3433 \\
OH group & 3433 & 3433 \\
$\mathrm{CH}$ stretching & 2853,2916 & 2853,2919 \\
$\mathrm{C}-\mathrm{N}$ stretching & 1695 & 1584 \\
$\mathrm{C}=\mathrm{C}$ quinoid ring & 1487 & 1491 \\
$\mathrm{C}=\mathrm{C}$ benzoid ring & 1633 & 1589 \\
Protonation of imine nitrogen & 1274 & 1295 \\
$\mathrm{CH}$ bending & 799 & 805 \\
$\mathrm{SO}_{3}{ }^{-}$group & 1079 & 1088 \\
$\mathrm{C}-\mathrm{S}$ vibration mode & 559 & 560 \\
\hline
\end{tabular}

TABLE 2

\begin{tabular}{lc}
\hline Wavelength $(\mathrm{nm})$ & Assignment \\
\hline $259-508$ & $\pi-\pi$ transition+localised Polaron \\
$>750$ & Delocalised polaron \\
$<259$ & Electronic conjugation due RGO \\
$<259$ & Chitosan absorption band \\
\hline
\end{tabular}

tially influence the sensor response [26]. For gold-based SPR sensor, the refractive index change of the gas phase was considered.

$$
s n=\frac{\Delta \theta}{\Delta n}
$$

where $\Delta \theta$ is the shift in the SPR angle (SPR shift) and $\Delta n$ is the refractive index change. 
TABLE 3: Optical constants values used in SPR studies.

\begin{tabular}{lcccc}
\hline Material & $\begin{array}{c}\text { Refractive index }(n) \\
(633 \mathrm{~nm})\end{array}$ & $\begin{array}{c}\text { Extinction coefficient }(k) \\
(633 \mathrm{~nm})\end{array}$ & $\begin{array}{c}\text { Real part dielectric constant } \\
(633 \mathrm{~nm})\end{array}$ & Ref. \\
\hline Dry air & 1.0026 & & 3.5853 & [65] \\
PANI/chitosan & 1.8957 & 0.0910 & 7.2599 & Equations $((1)-(12))$ \\
Ternary & 2.6973 & 0.1249 & - & Equations $((1)-(12))$ \\
Acetone vapour & 1.0011 & - & 3.1633 & {$[65]$} \\
SF11 glass prism & 1.7786 & $3.8652 \mathrm{E}-08$ & {$[77]$} \\
\hline
\end{tabular}

\section{Results and Discussion}

The successful synthesis of the composites was confirmed by FTIR and UV-VIS characterisations as shown in Figures 2 and 3, respectively. All the characteristics peaks for PANI, chitosan, and RGO were observed as summarised in Tables 1 and 2 for FTIR and UV-VIS, respectively. FTIR confirmed the presence of two important PANI peaks due to quinoid and benzoid vibration, respectively (Table 1) [71, 72].

Assignment of UV-VIS peaks in PANI/chitosan and ternary composites for UV-VIS, both composites, shows PANI characteristics' peaks attributable to $\pi-\pi^{*}$ transition of the benzenoid rings, localised polarons, and delocalised polarons at 325, 433, and above $900 \mathrm{~nm}$, respectively (Table 2). The broad peaks before $259 \mathrm{~nm}$ were all attributed to the presence of chitosan and RGO [73].

3.1. Optical Constants of the Sensing Layers and SPR Studies. The optical constants of the PANI composites, particularly the complex refractive index and the dielectric constants, were all evaluated through the application of equations $((1)-(12))$ at $633 \mathrm{~nm}$. Their values together with other materials used are illustrated in Table 3 . It is observed that the values of the optical constant in the ternary composites are greater compared to those of PANI/chitosan. This is due to the presence of reduced graphene oxide (RGO) which features many unique optical features [74-76].

Prior to the commencement of the SPR studies, different light sources were assessed for the applicability in SPR sensors based on the optical properties of the PANI composites. The assessment was conducted through the evaluation of penetration depth through the PANI composites (dielectric in this case) and the gold film. Table 4 shows the optical constant of gold thin film at different wavelengths of light [63].

For a better SPR sensing capability, the penetration depth through metal is required to be as low as possible while the penetration depth through the dielectric is required to be as high as possible. Figure 4 shows a comparison between $\left(\delta_{m}\right)$ and the composite materials $\left(\delta_{d}\right)$ at different light source wavelengths of red light $(633 \mathrm{~nm})$, yellow light $(589 \mathrm{~nm})$, green light $(546 \mathrm{~nm})$, violet light $(441 \mathrm{~nm})$, and blue light ( $436 \mathrm{~nm}, \mathrm{G}$-Line). Based on the above criterion, $633 \mathrm{~nm}$ was selected as the best light source for the subsequent SPR sensing studies based on PANI/chitosan and ternary composites. It can be observed that blue and violet light sources are not feasible as far as this study is concern.
TABle 4: Optical constant values of gold film at different wavelengths of light sources.

\begin{tabular}{lccc}
\hline $\begin{array}{l}\text { Wavelength } \\
(\mathrm{nm})\end{array}$ & $\begin{array}{c}\text { Refractive } \\
\text { index }(n)\end{array}$ & $\begin{array}{c}\text { Extinction } \\
\text { coefficient }(k)\end{array}$ & $\begin{array}{c}\text { Real part dielectric } \\
\text { constant }\end{array}$ \\
\hline 633 & 0.19404 & 3.5934 & -12.875 \\
589 & 0.26146 & 3.1019 & -9.5532 \\
546 & 0.39631 & 2.5581 & -6.3871 \\
441 & 1.55410 & 1.9847 & -1.5239 \\
436 & 1.57050 & 1.9933 & -1.5067 \\
\hline
\end{tabular}

However, apart from the red light source which is the best, yellow light could also be applicable.

In order to obtain maximum sensitivity in our studies, we optimized the reflectance minimum $\left(R_{\min }\right)$ and minimized the width of the resulting resonance curve. As shown in Figures 5(a) and 5(b), this was achieved by selecting the appropriate thickness of the gold layer. We selected 10 different thicknesses of gold, from $42 \mathrm{~nm}$ to $51 \mathrm{~nm}$ in dry air. The narrowest width of SPR curve is observed in $51 \mathrm{~nm}$ thickness. However, its reflectance minimum is the shallowest. But as shown in the inset of Figures 5(a) and 5(b), the greatest depth is observed when the thickness of the gold is decreased to $46 \mathrm{~nm}$ (Figure 5(b)). In addition, its full width at half maximum is not the worst. As such, we selected the appropriate thickness of the gold layer throughout this study as $46 \mathrm{~nm}$.

For the selection of appropriate thickness of the PANI layers, it is well-known that reflectance minimum and FWHM of SPR curves deteriorate with increasing thickness of over layer coatings $[26,74]$ and Figure 6 proves that for Figure 6(a), PANI/chitosan, and Figure 6(b), ternary composites films. However, in this study, thickness that could be achieved in reality has been considered $[77,78]$. As shown in the figures (Figures 6(a) and 6(b)), as the thickness increases from $10 \mathrm{~nm}$ to $16 \mathrm{~nm}$ in dry air, the properties tend to be affected significantly. But we assumed the attainment of $15 \mathrm{~nm}$ thickness of the PANI composites as something closer to reality. As such, all our evaluations were based on that thickness except otherwise described.

The response linearity of the proposed sensor to change in the refractive index is illustrated in Figure 7. The values were generated by increasing the refractive index of dry air at interval of 0.005 due to the presence of analyte. It is shown that the SPR shift is completely linear over the wide range of refractive index change for both the bare gold-based SPR 


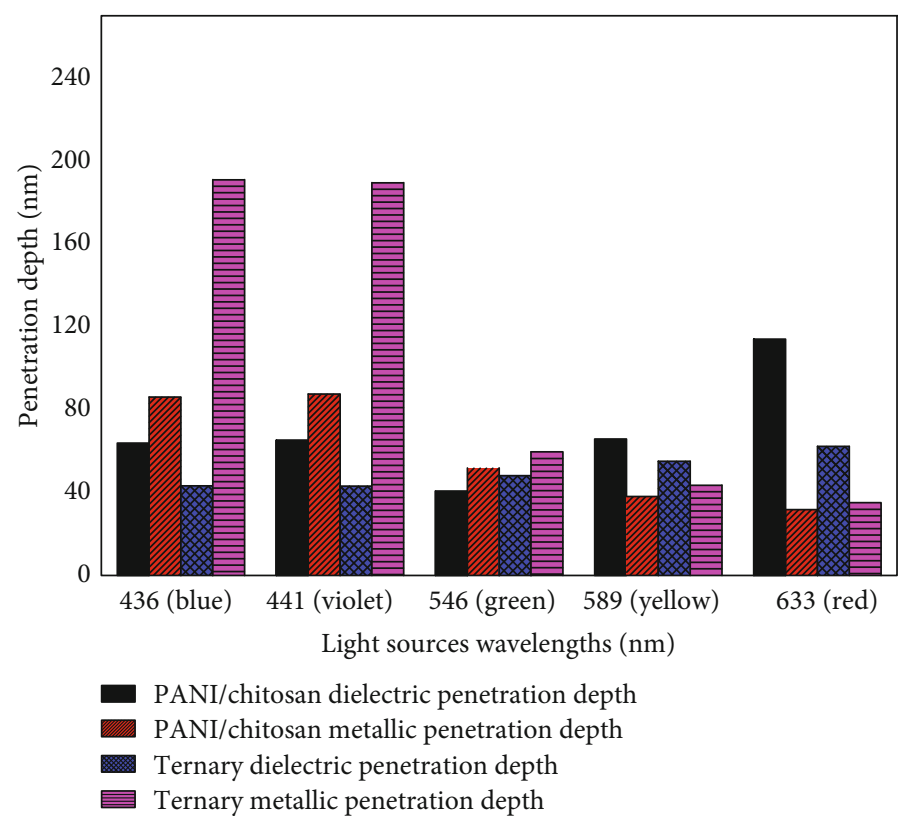

FIGURE 4: Comparison between metallic and dielectric penetration depth for PANI/chitosan and ternary composites at different wavelengths of light.

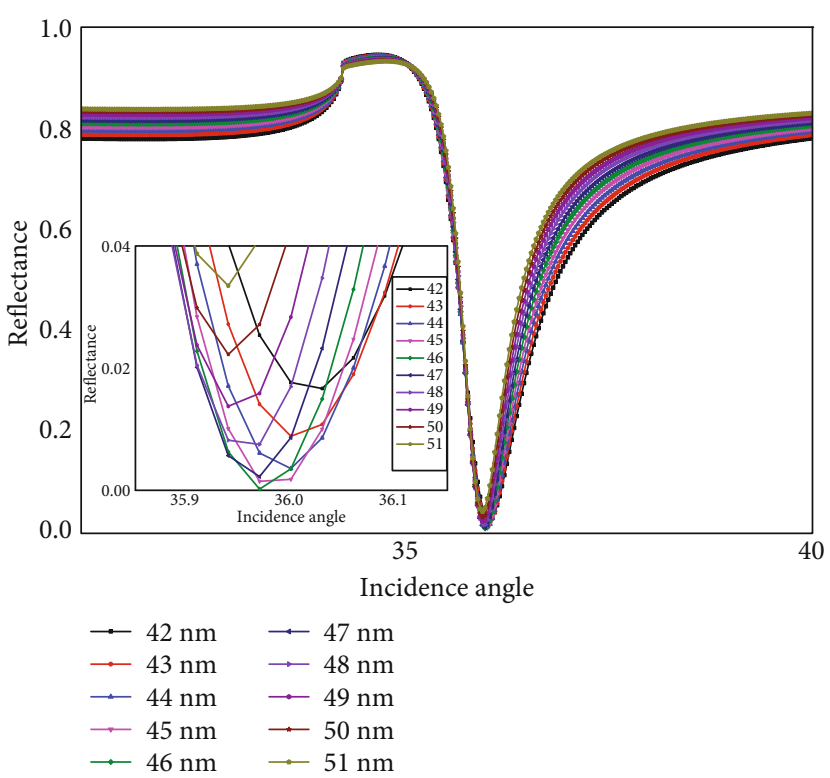

(a)

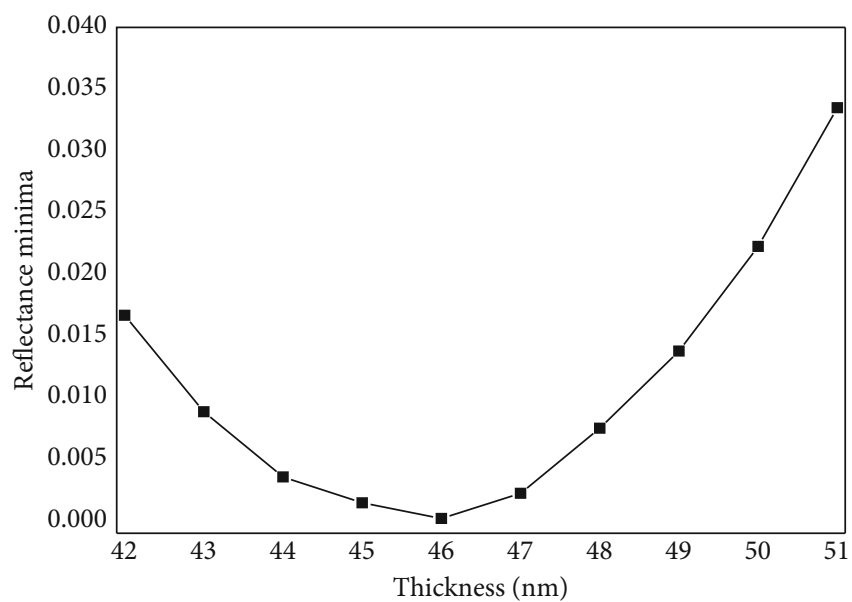

(b)

FIgURE 5: (a) Optimisation of the thickness of gold layer for SPR sensing in gas phase. The inset shows the thickness that gives the deepest reflectance minimum $(46 \mathrm{~nm}$ ). (b) Reflectance minima versus different thicknesses of gold layer.

sensor and the composite-based SPR sensors. This shows the potential application of the PANI composite materials in SPR sensors.

Figure 8 shows the SPR curves generated from the detection of low concentration of acetone vapour in air from $0.5 \mathrm{ppm}$ to $10 \mathrm{ppm}$ using gold-based (Figure $8(\mathrm{a})$ ), PANI/chitosan composite-based (Figure 8(b)), and ternary compositebased (Figure 8(c)) SPR sensors. Using equation (17), the refractive indices of acetone vapour at different concentrations were calculated. The values of refractive index change with respect to air are illustrated in Table 5. It is observed from Figure 8(a) that bare gold surface could not show any significant SPR shift for acetone vapour in $0.5 \mathrm{ppm}$ to $10 \mathrm{ppm}$ concentration range. This is the diabetes range of interest. However, the PANI/chitosan and the ternary composites show maximum sensitivity of about 60 and 180 degree per refractive index change, respectively. The performance of the gold-based SPR sensor in the detection of the acetone vapour is due to its lack of adsorption capability to gases at a low concentration. 


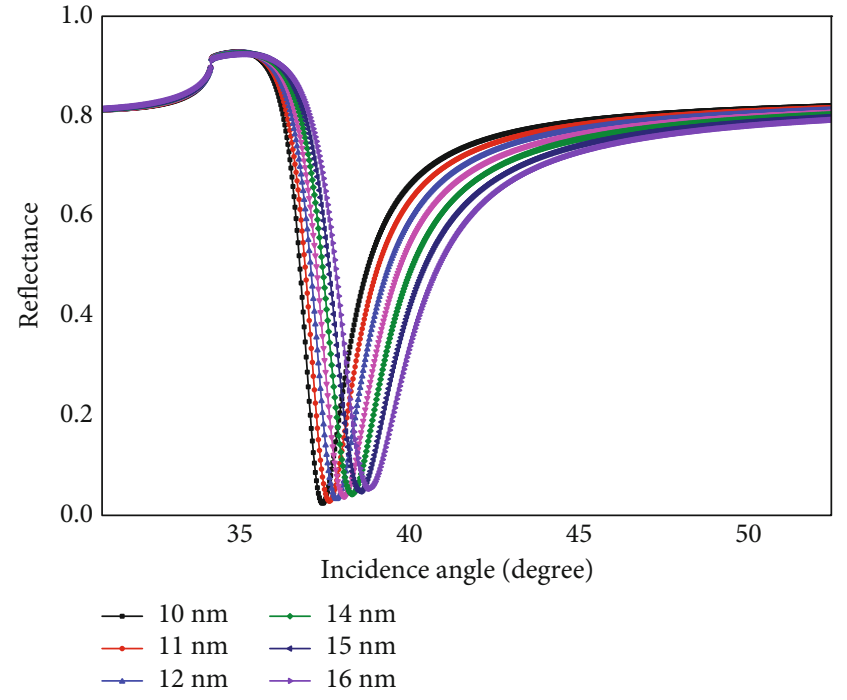

(a)

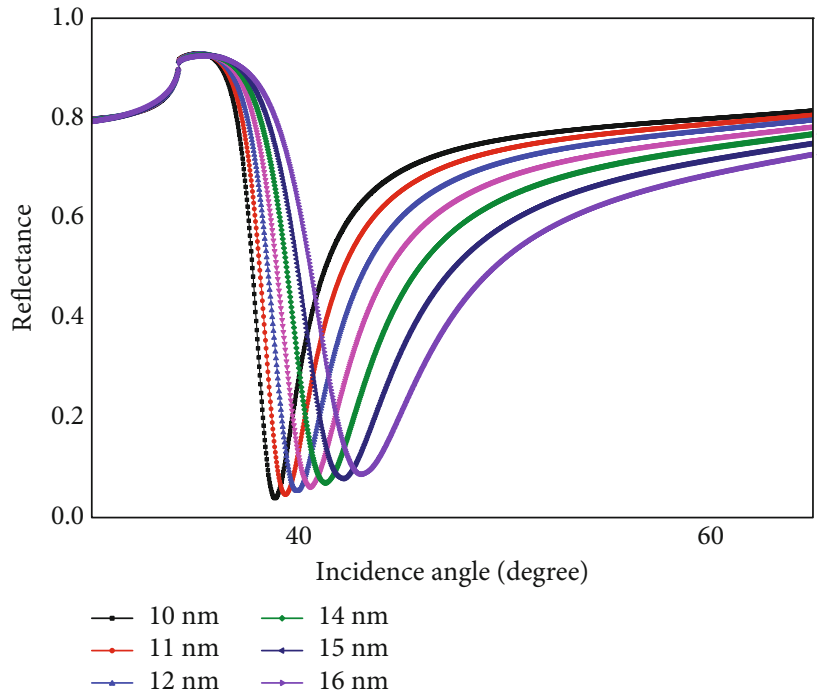

(b)

FIgure 6: Optimisation of the PANI composites' layer thickness for SPR sensing in gas phase in (a) PANI/chitosan and (b) ternary composites' films.

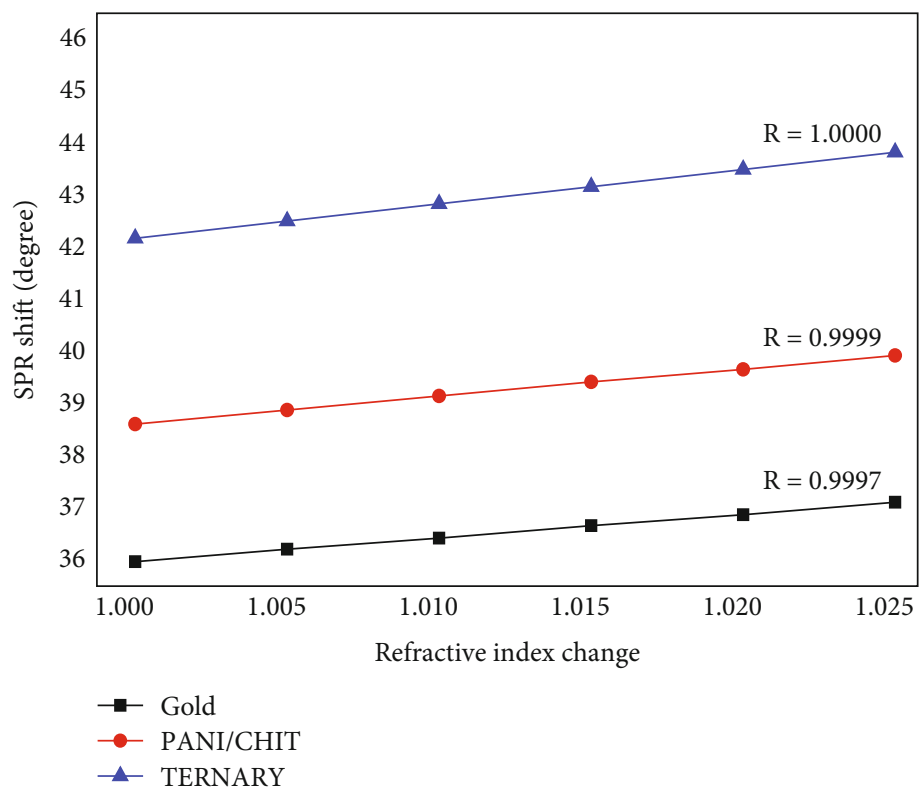

FIGURE 7: Comparison in terms of linearity in gas phase SPR sensing of R.I. variation in bare gold-, PANI/chitosan-, and ternary compositebased SPR biosensor with their correlation coefficients.

The selectivity of the PANI/chitosan- and the ternary composite-based SPR biosensors to acetone vapour was also investigated by examining the sensor response (SPR shift) to the common interfering vapours such as ethanol, methanol, and ammonia $[79,80]$. Figure $8(\mathrm{~d})$ shows the selectivity of the two sensing layers to $10 \mathrm{ppm}$ concentrations of acetone, ethanol, methanol, and ammonia vapours with their refractive index values estimated based on Arago-Biot mixing formula (equation (17)). The increment in refractive index for the vapours with respect to that of air (1.000263719) was found to be $8.22 \times 10^{-9}, 6.14 \times$ $10^{-9}, 3.23 \times 10^{-9}$, and $1.12 \times 10^{-9}$ for acetone, ethanol, methanol, and ammonia vapours, respectively. Both the PANI/chitosan- and the ternary-based SPR sensors show higher response for acetone vapour compared to other three vapours. Furthermore, PANI/chitosan-based SPR sensors show no response to $10 \mathrm{ppm}$ concentrations of methanol and ammonia. In addition, the ternary composite-based SPR sensor still maintained the highest response compared to the PANI/chitosan-based sensor. 


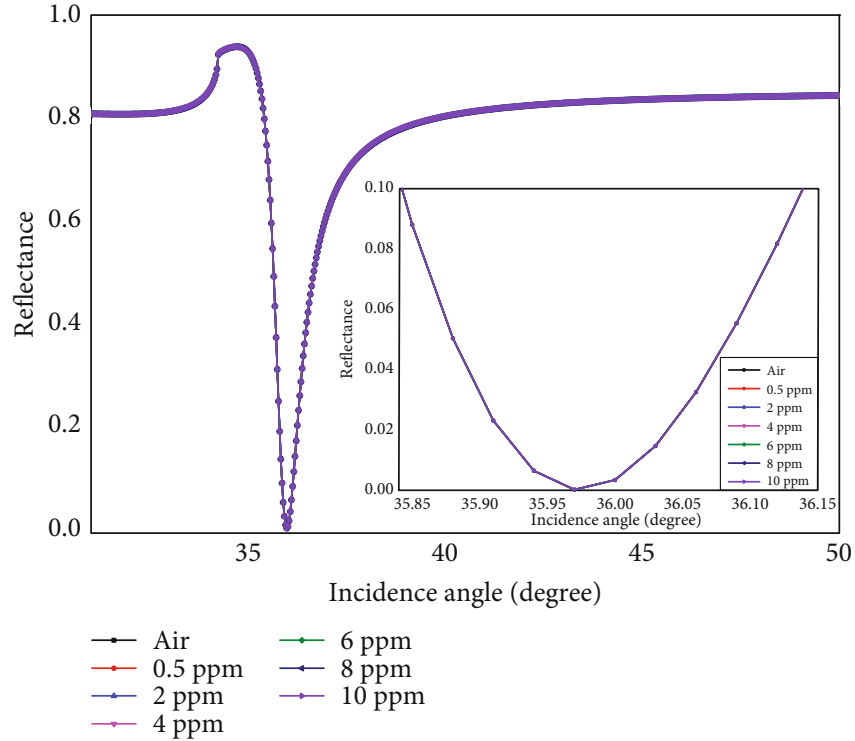

(a)

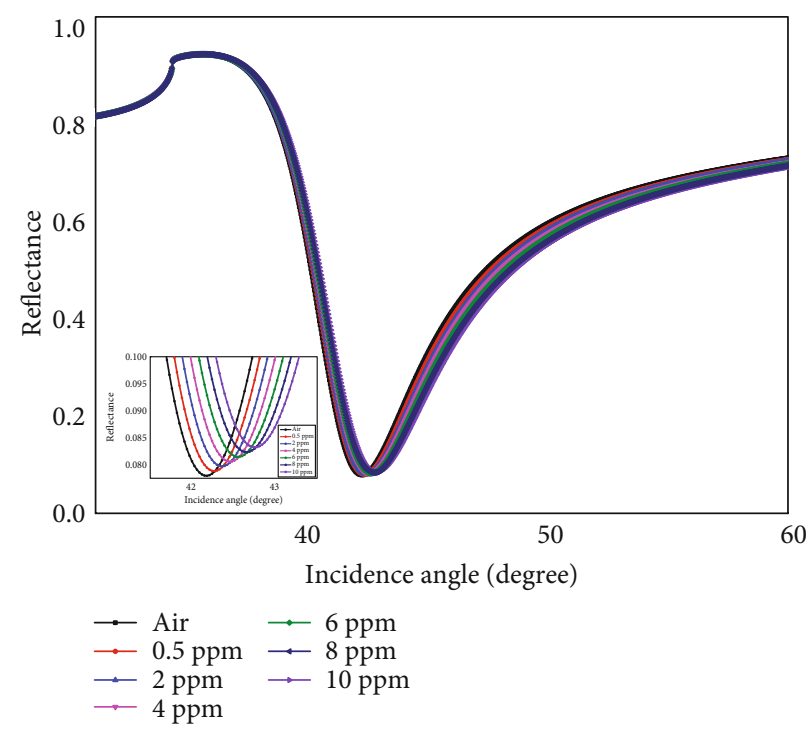

(c)

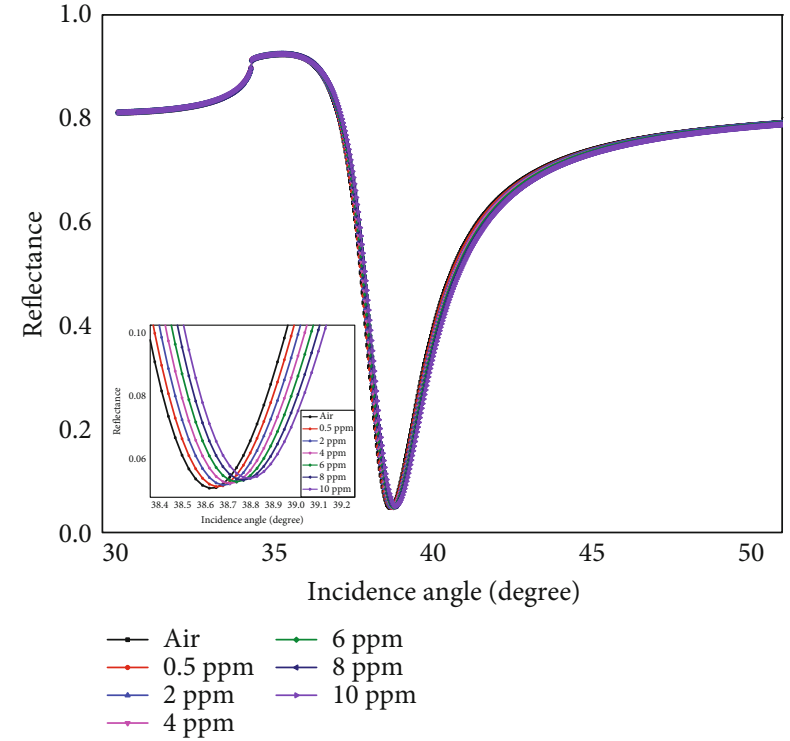

(b)

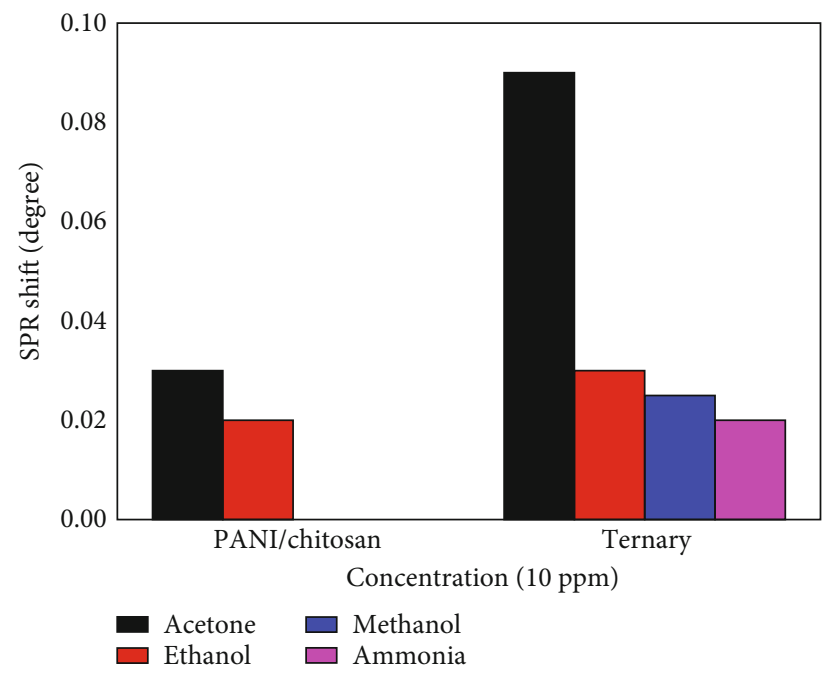

(d)

FIGURE 8: Gaseous-phase SPR sensing of acetone vapour using (a) bare gold-, (b) PANI/chitosan-, and (c) ternary composite-based SPR biosensor at different concentrations in ppm. (d) Selectivity of the SPR biosensor to acetone vapour against other interfering gases such as ethanol, methanol, and ammonia for $10 \mathrm{ppm}$ concentration.

The improved sensitivity and selectivity of PANI/chitosan- and the ternary composite-based SPR biosensors are due to the abundance of functional groups in the PANI composites, their possible solvation, and unique optical and electronic properties. In addition, the better performance of the ternary composites could also be attributed to the unique properties of reduced graphene oxide. Furthermore, graphene-based materials are known to adsorb carbonbased materials strongly and stably. Hence, our PANI composite-based SPR sensors, especially the ternary based, could open a way to achieve a reliable gas-phase SPR sensor with potential applications in health and environmental monitoring.
TABLE 5: Refractive index change of low-concentration acetone vapour in dry air (1.000263719, refractive index of acetone vapour at certain concentration) estimated using the Arago-Biot mixing formula.

\begin{tabular}{lc}
\hline Concentration $(\mathrm{ppm})$ & Refractive index change $(\Delta n)$ \\
\hline 0.5 & $4.11 \times 10^{-10}$ \\
2 & $1.64 \times 10^{-9}$ \\
4 & $3.29 \times 10^{-9}$ \\
6 & $4.93 \times 10^{-9}$ \\
8 & $6.58 \times 10^{-9}$ \\
10 & $8.22 \times 10^{-9}$ \\
\hline
\end{tabular}




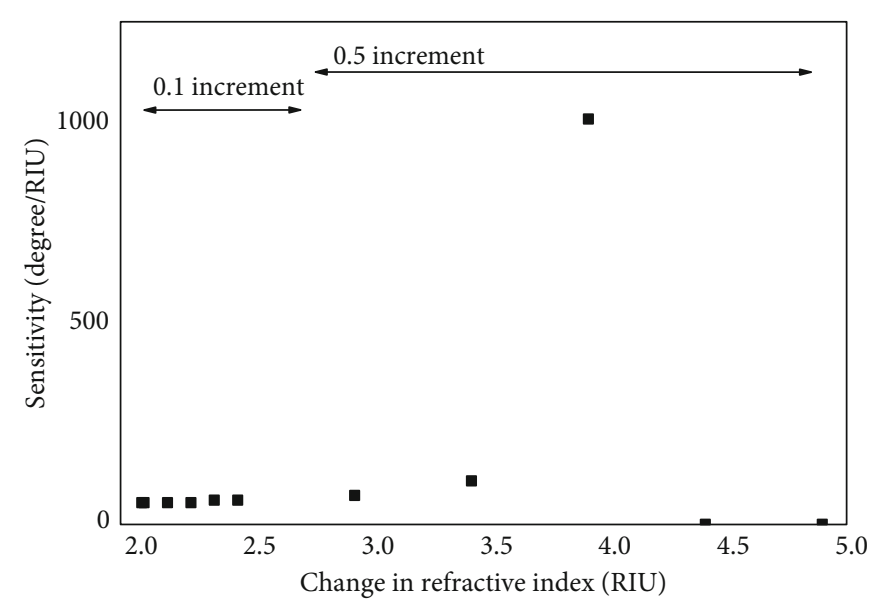

(a)

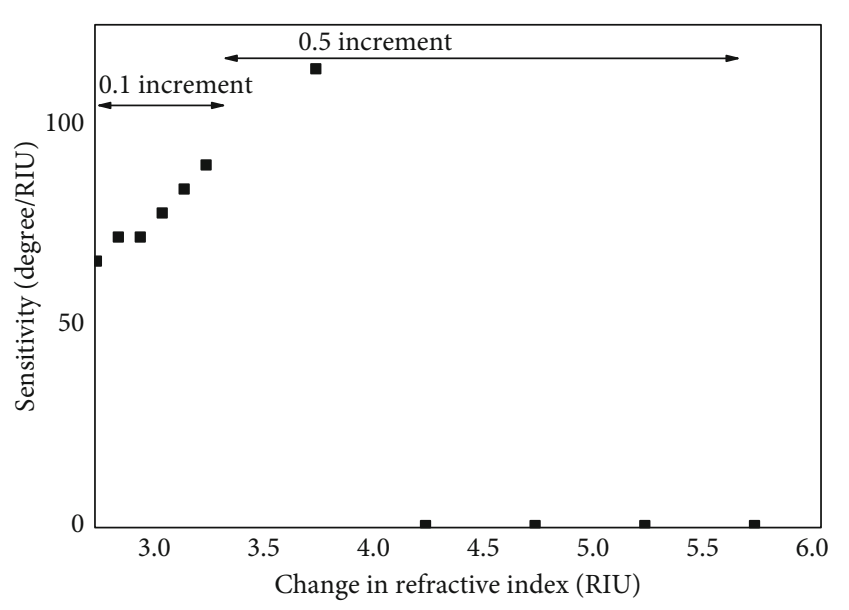

(b)

FIGURE 9: SPR shift due to improved refractive index of the sensing layers in (a) PANI/chitosan and (b) ternary composites.

Usually, gas sensing mechanisms are explained based on the change in the index of refraction in the analyte volume, the formation of an adsorbed layer, the diffusion of the analyte gas into the sensor layer, and the solvation of the analyte gas in the sensor layer [81]. Interestingly, in addition to low temperatures (below $100^{\circ} \mathrm{C}$ ), operation capability, and inexpensiveness in PANI-based sensors, PANI optical properties can be tailored (through doping or composites formation) to attract a specific gas (based on mechanisms stated in the introduction part) and thus can have high selectivity [54]. As such, we investigated the possible improvement in SPR shift with an increased value of refractive index in PANI/chitosan (Figure 9(a)) and ternary composites (Figure 9(b)). The imaginary value of the refractive index was kept constant. The result shows the 0.1 unit refractive increment to produce almost no improvement in SPR shift (also sensitivity) for both PANI/chitosan (Figure 9(a)) and ternary composites (Figure 9(b)). However, as the refractive index is increased by 0.5 units, the SPR shift increased significantly before dropping after adding about 1.5 unit of refractive index. Therefore, it could be concluded that the two composites need no further improvement. This is because the increment in refractive index that is normally achieved experimentally is within 0.1 unit increment range.

\section{Conclusions}

This work presents the application of PANI/chitosan composite and a ternary composite comprising of PANI, chitosan, and reduced graphene oxide in the SPR sensing of acetone vapour for the monitoring and screening of diabetes using mathematical modeling and simulation. The synthesis of the composites was based on oxidative polymerisation of aniline using ammonium peroxydisulfate (APS) and characterised using FTIR and UV-visible spectroscopy. Optical constants of the composites were extracted from the absorption spectra of the UV-VIS spectroscopy and applied in the simulation of a surface plasmon resonance- (SPR-) based acetone vapour sensor functionalised with PANI/chitosan and ternary composites for diabetes interest, $1.8 \mathrm{ppm}-$ $5.0 \mathrm{ppm}$. The response in the case of ternary composite shows best linearity with correlation coefficient values of $1.0 \mathrm{com}$ pared to PANI/chitosan- and gold-based SPR layers with 0.9999 and 0.9997 , respectively. The result indicates promising application of the composite-based sensor in the detection of acetone vapour at a low concentration down to less than $0.5 \mathrm{ppm}$. The maximum sensitivity values of about 60 and 180 degree per refractive index change were observed for PANI/chitosan and ternary composite sensing layers, respectively, in comparison with the bare goldbased SPR which shows zero response up to $10 \mathrm{ppm}$ concentration of acetone vapour in air. The sensing layers also show better selectivity to acetone vapour compared to ethanol, methanol, and ammonia vapours. Hence, the $\mathrm{PANI} /$ chitosan and ternary composite sensing layers, especially the ternary composite, would have a great potential in realising a gas-phase SPR biosensor for health and environmental monitoring.

\section{Data Availability}

Data would be made available upon request.

\section{Conflicts of Interest}

The authors declare no conflict of interests.

\section{Acknowledgments}

This work is supported by the Fundamental Research Grant Scheme, under Grant 015MA0-018 of the Ministry of Higher Education Malaysia and the Yayasan Universiti Teknologi PETRONAS-Fundamental Research Grant, under Grant 0153AA-E40.

\section{References}

[1] J. L. Santos and F. Farahi, Handbook of Optical Sensors, Crc Press, 2014. 
[2] A. K. Sharma and B. D. Gupta, "On the performance of different bimetallic combinations in surface plasmon resonance based fiber optic sensors," Journal of Applied Physics, vol. 101, no. 9, article 093111, 2007.

[3] R. P. Kooyman, "Physics of surface plasmon resonance," in Handbook of Surface Plasmon Resonance, p. 1, The Royal Society of Chemistry, 2008.

[4] J.-F. Masson, "Surface plasmon resonance clinical biosensors for medical diagnostics," ACS Sensors, vol. 2, no. 1, pp. 1630, 2017.

[5] L. Wu, H. Chu, W. Koh, and E. Li, "Highly sensitive graphene biosensors based on surface plasmon resonance," Optics Express, vol. 18, no. 14, pp. 14395-14400, 2010.

[6] J. Schwank, "Catalytic gold," Gold Bulletin, vol. 16, no. 4, pp. 103-110, 1983.

[7] M. E. H. Amrani, P. A. Payne, R. M. Dowdeswell, and A. D. Hoffman, "Frequency counting interrogation techniques applied to gas sensor arrays," Sensors and Actuators B: Chemical, vol. 57, no. 1-3, pp. 75-82, 1999.

[8] M. Erdoğan, R. Capan, and F. Davis, "Swelling behaviour of calixarene film exposed to various organic vapours by surface plasmon resonance technique," Sensors and Actuators B: Chemical, vol. 145, no. 1, pp. 66-70, 2010.

[9] K. C. Persaud, S. M. Khaffaf, P. J. Hobbs, and R. W. Sneath, "Assessment of conducting polymer odour sensors for agricultural malodour measurements," Chemical Senses, vol. 21, no. 5, pp. 495-505, 1996.

[10] P. Španěl, K. Dryahina, and D. Smith, “Acetone, ammonia and hydrogen cyanide in exhaled breath of several volunteers aged 4-83 years," Journal of Breath Research, vol. 1, no. 1, article $011001,2007$.

[11] T. H. Tranah, G. K. M. Vijay, J. M. Ryan, and D. L. Shawcross, "Systemic inflammation and ammonia in hepatic encephalopathy," Metabolic Brain Disease, vol. 28, no. 1, pp. 1-5, 2013.

[12] P. Španěl and D. Smith, "What is the real utility of breath ammonia concentration measurements in medicine and physiology?," Journal of Breath Research, vol. 12, no. 2, article 027102, 2018.

[13] V. Saasa, T. Malwela, M. Beukes, M. Mokgotho, C.-P. Liu, and B. Mwakikunga, "Sensing technologies for detection of acetone in human breath for diabetes diagnosis and monitoring," Diagnostics, vol. 8, no. 1, p. 12, 2018.

[14] D. Wang, Q. Zhang, M. R. Hossain, and M. Johnson, "High sensitive breath sensor based on nanostructured $\mathrm{K}_{2} \mathrm{~W}_{7} \mathrm{O}_{22}$ for detection of type 1 diabetes," IEEE Sensors Journal, vol. 18, no. 11, pp. 4399-4404, 2018.

[15] Z. Y. Gong, M. X. Sun, C. Y. Jian et al., “A ringdown breath acetone analyzer: performance and validation using gas chromatography-mass spectrometry," Journal of Analytical \& Bioanalytical Techniques, vol. S7, no. 12, p. 013, 2014.

[16] D. Guo, D. Zhang, L. Zhang, and G. Lu, "Non-invasive blood glucose monitoring for diabetics by means of breath signal analysis," Sensors and Actuators B: Chemical, vol. 173, pp. 106-113, 2012.

[17] A. Rydosz, "Microsystem in LTCC technology to the detection of acetone in exhaled breath," International Journal of Information and Electronics Engineering, vol. 5, no. 2, pp. 98101, 2015.

[18] I. Ueta, Y. Saito, M. Hosoe et al., "Breath acetone analysis with miniaturized sample preparation device: In-needle preconcentration and subsequent determination by gas chromatography-mass spectroscopy," Journal of Chromatography $B$, vol. 877, no. 24, pp. 2551-2556, 2009.

[19] M. Narjinary, P. Rana, A. Sen, and M. Pal, "Enhanced and selective acetone sensing properties of $\mathrm{SnO}_{2}-\mathrm{MWCNT}$ nanocomposites: promising materials for diabetes sensor," Materials \& Design, vol. 115, pp. 158-164, 2017.

[20] I. Fratoddi, I. Venditti, C. Cametti, and M. V. Russo, "Chemiresistive polyaniline-based gas sensors: a mini review," Sensors and Actuators B: Chemical, vol. 220, pp. 534-548, 2015.

[21] H. Bai and G. Shi, "Gas sensors based on conducting polymers," Sensors, vol. 7, no. 3, pp. 267-307, 2007.

[22] S. Wei, G. Zhao, W. Du, and Q. Tian, "Synthesis and excellent acetone sensing properties of porous $\mathrm{WO}_{3}$ nanofibers," Vacuum, vol. 124, pp. 32-39, 2016.

[23] Z. Liu, B. Liu, W. Xie et al., "Enhanced selective acetone sensing characteristics based on co-doped $\mathrm{WO}_{3}$ hierarchical flower-like nanostructures assembled with nanoplates," Sensors and Actuators B: Chemical, vol. 235, pp. 614-621, 2016.

[24] J. Shi, G. Hu, Y. Sun et al., " $\mathrm{WO}_{3}$ nanocrystals: synthesis and application in highly sensitive detection of acetone," Sensors and Actuators B: Chemical, vol. 156, no. 2, pp. 820-824, 2011.

[25] S. V. Ryabtsev, A. V. Shaposhnick, A. N. Lukin, and E. P. Domashevskaya, "Application of semiconductor gas sensors for medical diagnostics," Sensors and Actuators B: Chemical, vol. 59, no. 1, pp. 26-29, 1999.

[26] N. Menegazzo, B. Herbert, S. Banerji, and K. S. Booksh, "Discourse on the utilization of polyaniline coatings for surface plasmon resonance sensing of ammonia vapor," Talanta, vol. 85, no. 3, pp. 1369-1375, 2011.

[27] B. C. Sih, M. O. Wolf, D. Jarvis, and J. F. Young, "Surfaceplasmon resonance sensing of alcohol with electrodeposited polythiophene and gold nanoparticle-oligothiophene films," Journal of Applied Physics, vol. 98, no. 11, article 114314, 2005.

[28] S. Şen, R. Capan, M. Özel, A. Hassan, H. Namli, and O. Turhan, "P-Phthalimidobenzoic thin film for volatile organic vapor detection," Journal of Optoelectronics and Advanced Materials, vol. 12, article 1534, 2010.

[29] R. Capan, A. K. Ray, A. K. Hassan, and T. Tanrisever, "Poly (methyl methacrylate) films for organic vapour sensing," Journal of Physics D: Applied Physics, vol. 36, no. 9, pp. 1115-1119, 2003.

[30] M. Erdoğan, I. Capan, C. Tarimci, and A. Hassan, "Modeling of vapor sorption in polymeric film studied by surface plasmon resonance spectroscopy," Journal of Colloid and Interface Science, vol. 323, no. 2, pp. 235-241, 2008.

[31] S. Nengsih, A. A. Umar, M. M. Salleh, and M. Yahaya, "Detection of volatile organic compound gas using localized surface plasmon resonance of gold nanoparticles," Sains Malaysiana, vol. 40, pp. 231-235, 2011.

[32] A. A. Alwahib, A. R. Sadrolhosseini, M. N. An'amt et al., "Reduced graphene oxide/maghemite nanocomposite for detection of hydrocarbon vapor using surface plasmon resonance," IEEE Photonics Journal, vol. 8, no. 4, pp. 1-9, 2016.

[33] J. Matsui, K. Akamatsu, N. Hara et al., "SPR sensor chip for detection of small molecules using molecularly imprinted polymer with embedded gold nanoparticles," Analytical Chemistry, vol. 77, no. 13, pp. 4282-4285, 2005.

[34] V. Amendola, R. Pilot, M. Frasconi, O. M. Maragò, and M. A. Iatì, "Surface plasmon resonance in gold nanoparticles: a 
review," Journal of Physics: Condensed Matter, vol. 29, no. 20, article 203002, 2017.

[35] Q. Li, Q. Wang, X. Yang, K. Wang, H. Zhang, and W. Nie, "High sensitivity surface plasmon resonance biosensor for detection of microRNA and small molecule based on graphene oxide-gold nanoparticles composites," Talanta, vol. 174, pp. 521-526, 2017.

[36] S. A. Zynio, A. V. Samoylov, E. R. Surovtseva, V. M. Mirsky, and Y. M. Shirshov, "Bimetallic layers increase sensitivity of affinity sensors based on surface plasmon resonance," Sensors, vol. 2, no. 2, pp. 62-70, 2002.

[37] T. Srivastava, R. Jha, and R. Das, "High-performance bimetallic SPR sensor based on periodic-multilayer-waveguides," IEEE Photonics Technology Letters, vol. 23, no. 20, pp. 14481450, 2011.

[38] W. Du and F. Zhao, "Silicon carbide based surface plasmon resonance waveguide sensor with a bimetallic layer for improved sensitivity," Materials Letters, vol. 186, pp. 224226, 2017.

[39] S. H. Kim, T. U. Kim, H. Y. Jung, H. C. Ki, D. G. Kim, and B.-T. Lee, "The effect of Au/Ag bimetallic thin-films on surface plasmon resonance properties comparing with those of $\mathrm{Au}$ and Ag single thin-films," Journal of Nanoscience and Nanotechnology, vol. 18, no. 3, pp. 1777-1781, 2018.

[40] L. Wang, X.-M. Wan, R. Gao, D.-F. Lu, and Z.-M. Qi, "Nanoporous gold films prepared by a combination of sputtering and dealloying for trace detection of benzo [a] pyrene based on surface plasmon resonance spectroscopy," Sensors, vol. 17, no. 6, p. 1255, 2017.

[41] C. G. Artur and W.-C. Shih, "Nanoporous gold nanoparticles and arrays for label-free nanoplasmonic biosensing," in Miniature Fluidic Devices for Rapid Biological Detection, pp. 25-67, Springer, 2018.

[42] X. Lang, L. Qian, P. Guan, J. Zi, and M. Chen, "Localized surface plasmon resonance of nanoporous gold," Applied Physics Letters, vol. 98, no. 9, article 093701, 2011.

[43] D. van Noort and C.-F. Mandenius, "Porous gold surfaces for biosensor applications," Biosensors and Bioelectronics, vol. 15, no. 3-4, pp. 203-209, 2000.

[44] S. Szunerits, N. Maalouli, E. Wijaya, J.-P. Vilcot, and R. Boukherroub, "Recent advances in the development of graphene-based surface plasmon resonance (SPR) interfaces," Analytical and Bioanalytical Chemistry, vol. 405, no. 5, pp. 1435-1443, 2013.

[45] N. E. Agbor, M. C. Petty, and A. P. Monkman, "Polyaniline thin films for gas sensing," Sensors and Actuators B: Chemical, vol. 28, no. 3, pp. 173-179, 1995.

[46] E. Yılmaz, E. Özgür, N. Bereli, D. Türkmen, and A. Denizli, "Plastic antibody based surface plasmon resonance nanosensors for selective atrazine detection," Materials Science and Engineering: C, vol. 73, pp. 603-610, 2017.

[47] K. Shah, N. K. Sharma, and V. Sajal, "SPR based fiber optic sensor with bi layers of indium tin oxide and platinum: a theoretical evaluation," Optik, vol. 135, pp. 50-56, 2017.

[48] G. An, S. Li, H. Wang, and X. Zhang, "Metal oxide-graphenebased quasi-D-shaped optical fiber plasmonic biosensor," IEEE Photonics Journal, vol. 9, no. 4, pp. 1-9, 2017.

[49] C. Nylander, M. Armgarth, and I. Lundström, "An ammonia detector based on a conducting polymer," in Anal. Chem. Symp. Ser, pp. 203-207, Fukuoka, Japan, 1983.
[50] T. I. Nasution, I. Nainggolan, S. D. Hutagalung, K. R. Ahmad, and Z. A. Ahmad, "The sensing mechanism and detection of low concentration acetone using chitosan-based sensors," Sensors and Actuators B: Chemical, vol. 177, pp. 522-528, 2013.

[51] O. Salihoglu, S. Balci, and C. Kocabas, "Plasmon-polaritons on graphene-metal surface and their use in biosensors," Applied Physics Letters, vol. 100, no. 21, article 213110, 2012.

[52] S. Zeng, S. Hu, J. Xia et al., "Graphene- $\mathrm{MoS}_{2}$ hybrid nanostructures enhanced surface plasmon resonance biosensors," Sensors and Actuators B: Chemical, vol. 207, pp. 801-810, 2015.

[53] Y. Zhao, X.-g. Li, X. Zhou, and Y.-n. Zhang, "Review on the graphene based optical fiber chemical and biological sensors," Sensors and Actuators B: Chemical, vol. 231, pp. 324-340, 2016.

[54] K. M. E. Stewart, Design of polymeric sensing materials for volatile organic compounds: optimized material selection for ethanol with mechanistic explanations 2016, https:// wileyonlinelibrary.com.

[55] J. Brandrup, E. H. Immergut, E. A. Grulke, A. Abe, and D. R. Bloch, Polymer Handbook, vol. 89, Wiley, New York, 1999.

[56] B. Song, D. Li, W. Qi, M. Elstner, C. Fan, and H. Fang, "Graphene on $\mathrm{Au}(111)$ : a highly conductive material with excellent adsorption properties for high-resolution bio/nanodetection and identification," ChemPhysChem, vol. 11, no. 3, pp. 585-589, 2010.

[57] V. Janaki, B. T. Oh, K. Shanthi, K. J. Lee, A. K. Ramasamy, and S. Kamala-Kannan, "Polyaniline/chitosan composite: an ecofriendly polymer for enhanced removal of dyes from aqueous solution," Synthetic Metals, vol. 162, no. 11-12, pp. 974-980, 2012.

[58] T. Thanpitcha, A. Sirivat, A. M. Jamieson, and R. Rujiravanit, "Preparation and characterization of polyaniline/chitosan blend film," Carbohydrate Polymers, vol. 64, no. 4, pp. 560 568, 2006.

[59] R. Kabiri and H. Namazi, "Synthesis of cellulose/reduced graphene oxide/polyaniline nanocomposite and its properties," International Journal of Polymeric Materials and Polymeric Biomaterials, vol. 65, no. 13, pp. 675-682, 2016.

[60] V. H. Nguyen, C. Lamiel, D. Kharismadewi, V. C. Tran, and J. J. Shim, "Covalently bonded reduced graphene oxide/polyaniline composite for electrochemical sensors and capacitors," Journal of Electroanalytical Chemistry, vol. 758, pp. 148-155, 2015.

[61] M. Fox, Optical properties of solids, AAPT, 2002.

[62] S. B. Aziz, A. Q. Hassan, S. J. Mohammed et al., "Structural and optical characteristics of PVA: C-dot composites: tuning the absorption of ultra violet (UV) region," Nanomaterials, vol. 9, no. 2, p. 216, 2019.

[63] D. I. Yakubovsky, A. V. Arsenin, Y. V. Stebunov, D. Y. Fedyanin, and V. S. Volkov, "Optical constants and structural properties of thin gold films," Optics Express, vol. 25, no. 21, pp. 25574-25587, 2017.

[64] M. N. Polyanskiy, "Refractive index database," May 2019, https://refractiveindex.info.

[65] G. W. C. Kaye and T. H. Laby, Tables of physical and chemical constants and some mathematical functions, Longmans, Green, and Company, 1911.

[66] G. Kaye and T. Laby, Tables of physical and chemical constants, 1973, Longman, London, 1989.

[67] G. Baranović, "Refractive index mixing rules and excess infrared spectra of binary mixtures," Applied Spectroscopy, vol. 71, no. 5, pp. 1039-1049, 2017. 
[68] L. Lizarraga, E. María Andrade, and F. Victor Molina, "Swelling and volume changes of polyaniline upon redox switching," Journal of Electroanalytical Chemistry, vol. 561, pp. 127-135, 2004.

[69] J. G. Varghese, A. A. Kittur, P. S. Rachipudi, and M. Y. Kariduraganavar, "Synthesis, characterization and pervaporation performance of chitosan-g- polyaniline membranes for the dehydration of isopropanol," Journal of Membrane Science, vol. 364, no. 1-2, pp. 111-121, 2010.

[70] S. J. Kim, S. G. Yoon, I. Y. Kim, and S. I. Kim, "Swelling characterization of the semiinterpenetrating polymer network hydrogels composed of chitosan and poly (diallyldimethylammonium chloride)," Journal of Applied Polymer Science, vol. 91, no. 5, pp. 2876-2880, 2004.

[71] A. M. Solonaru and M. Grigoras, "Water-soluble polyaniline/graphene composites as materials for energy storage applications," Express Polymer Letters, vol. 11, no. 2, pp. 127-139, 2017.

[72] S. Sultana, N. Ahmad, S. M. Faisal, M. Owais, and S. Sabir, "Synthesis, characterisation and potential applications of polyaniline/chitosan-Ag-nano-biocomposite," IET Nanobiotechnology, vol. 11, no. 7, pp. 835-842, 2017.

[73] L. Dennany, P. Innis, S. McGovern, G. G. Wallace, and R. J. Forster, "Electronic interactions within composites of polyanilines formed under acidic and alkaline conditions. Conductivity, ESR, Raman, UV-vis and fluorescence studies," Physical Chemistry Chemical Physics, vol. 13, no. 8, pp. 3303-3310, 2011.

[74] S. H. Choi, Y. L. Kim, and K. M. Byun, "Graphene-on-silver substrates for sensitive surface plasmon resonance imaging biosensors," Optics Express, vol. 19, no. 2, pp. 458-466, 2011.

[75] C. Gómez-Navarro, R. T. Weitz, A. M. Bittner et al., "Electronic transport properties of individual chemically reduced graphene oxide sheets," Nano Letters, vol. 7, no. 11, pp. 3499-3503, 2007.

[76] F. Bonaccorso, Z. Sun, T. Hasan, and A. C. Ferrari, "Graphene photonics and optoelectronics," Nature Photonics, vol. 4, no. 9, pp. 611-622, 2010.

[77] B. Wessling, "New insight into organic metal polyaniline morphology and structure," Polymers, vol. 2, no. 4, pp. 786$798,2010$.

[78] B. Wessling, Dispersions of intrinsically conductive polymers, and methods for the production thereof, Google Patents, 2010.

[79] F. Usman, J. O. Dennis, A. Y. Ahmed, F. Meriaudeau, O. B. Ayodele, and A. A. S. Rabih, "A review of biosensors for non-invasive diabetes monitoring and screening in human exhaled breath," IEEE Access, vol. 7, pp. 5963-5974, 2018.

[80] A. A. S. Rabih, J. O. Dennis, A. Y. Ahmed et al., "MEMS-based acetone vapor sensor for non-invasive screening of diabetes," IEEE Sensors Journal, vol. 18, no. 23, pp. 9486-9500, 2018.

[81] A. Nooke, Gas detection by means of surface plasmon resonance enhanced ellipsometry, BAM Bundesanstalt für Materialforschung und -prüfung, 2012. 


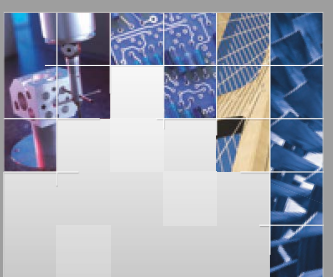

\section{Enfincering}
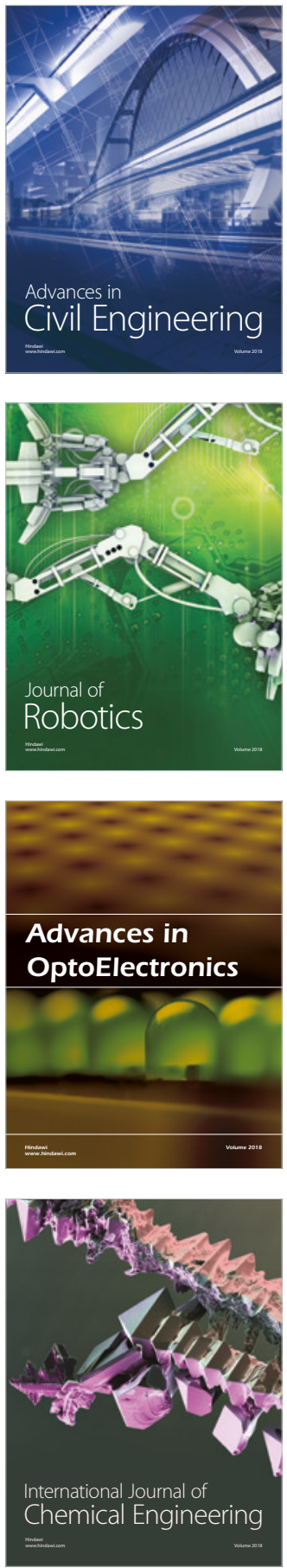

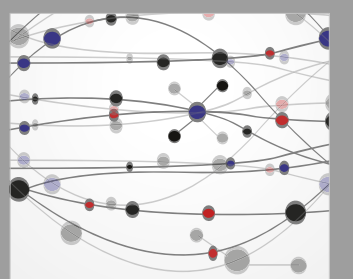

\section{Rotating \\ Machinery}

The Scientific World Journal

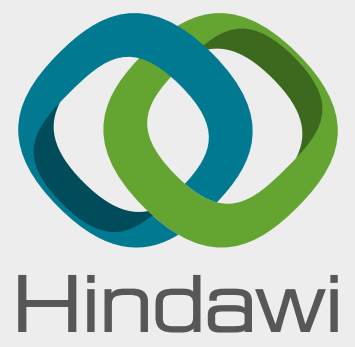

Submit your manuscripts at

www.hindawi.com
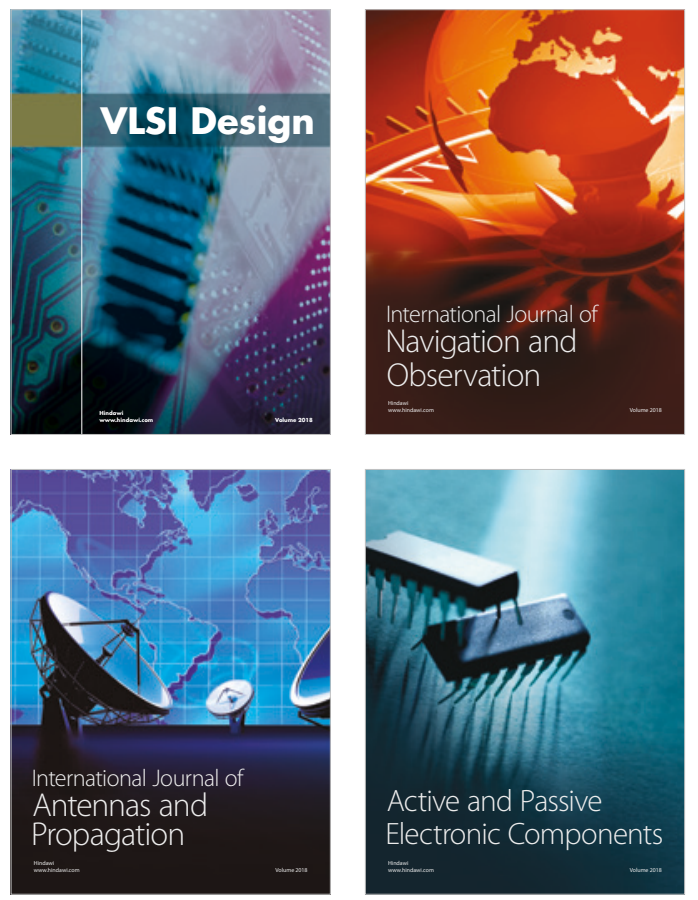
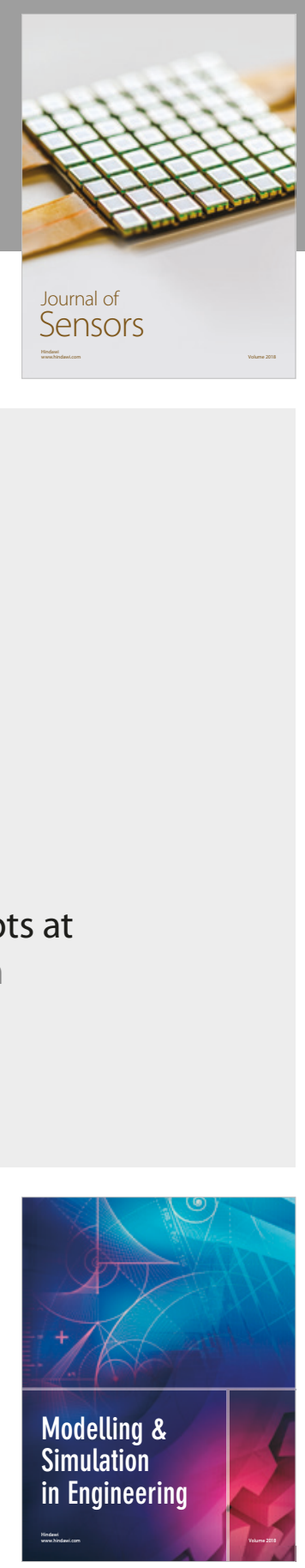

\section{Advances \\ Multimedia}
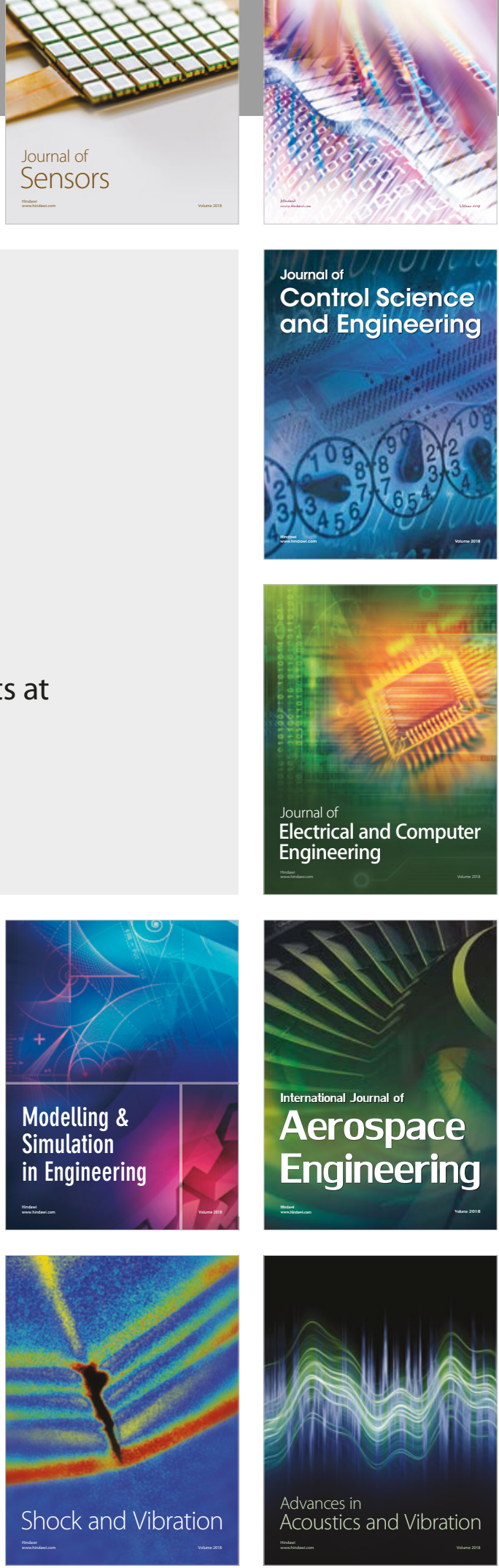